\title{
Self-phase modulation in chirped-pulse amplification
}

\author{
M. D. Perry, T. Ditmire, and B. C. Stuart \\ Laser Program, Lawrence Livermore National Laboratory, P.O. Box 808, L-493, Livermore, California 94550
}

Received August 2, 1994

\begin{abstract}
The effect of self-phase modulation in chirped-pulse amplification is investigated. A numerical model is used to predict the effects of phase modulation on pulse recompression, and experimental results are presented that agree well with the calculations. We show that even moderate self-phase modulation can significantly distort the recompressed pulse after amplification, thereby reducing the peak power and degrading the pulse contrast.
\end{abstract}

Table-top, high-irradiance laser systems that use the technique of chirped-pulse amplification ${ }^{1}$ (CPA) have proliferated dramatically. ${ }^{2}$ This technique has made possible the amplification of very short laser pulses ( $\leq 1 \mathrm{ps})$ to very high peak powers $\left(\geq 10^{12} \mathrm{~W}\right)$ in small-scale laser systems. To avoid damaging nonlinear effects such as self-focusing and self-phase modulation, which normally would be associated with the propagation of such high-power laser pulses through a laser amplifier chain, a short broadband pulse is initially stretched by several thousand by inducing a large amount of group-velocity dispersion with a grating pair. ${ }^{3,4}$ After amplification the pulse is compressed to near its initial duration with a second grating pair. ${ }^{5}$

A multitude of factors can degrade the pulse on compression. They can be grouped into those that affect the pulse amplitude (gain narrowing, ${ }^{6}$ amplitude modulation from étalons or spectral filters, saturated gain ${ }^{7}$ ) and those that affect its phase (such as material dispersion and uncompensated cubic or quartic phase). ${ }^{8}$ To varying degrees, these effects can be minimized by different stretcher/compressor designs..$^{9,10}$ In this Letter we show that self-phase modulation of the stretched pulse as it propagates through the amplifier chain will ultimately limit the peak power and pulse contrast achievable on recompression.

In CPA systems the effect of self-phase modulation on the pulse spectrum is small. However, recompression of the amplified, stretched pulses can be seriously affected by phase modulation. ${ }^{7,11}$

To examine this effect, we developed a simple propagation model in which the transverse intensity distribution is coupled to the temporal evolution of the pulse only by providing a spatially varying nonlinear phase, defined by

$$
B(\mathbf{r}, t)=\frac{2 \pi}{\lambda} \int n_{2}|E(\mathbf{r}, t)|^{2} \mathrm{~d} z,
$$

where $n_{2}$ is the nonlinear component of the refractive index, $n=n_{0}+n_{2}|E(\mathbf{r}, t)|^{2}$. Specifically the field is separable into a transverse distribution, $F(x, y)$, and a slowly varying complex amplitude, $A(z, t)$, such that $E(\mathbf{r}, t)=\left\{F(x, y) A(z, t) \exp \left[i\left(k_{0} z-\omega_{0} t\right)\right]+\right.$ c.c. $\} / 2$.

We assume that the initial low-energy pulse is transform limited with a field distribution, $E_{0}(z, t)$, and a Fourier transform, $E_{0}\left(z, \omega-\omega_{0}\right)$. This pulse is stretched by a grating-based stretcher with a phase function, $\phi_{\mathrm{str}}(\omega)$. An amplitude transfer function, $M(\omega)$, can be included to account for any spectral filtering in the pulse stretcher:

$$
\begin{aligned}
E_{\mathrm{str}}\left(\mathbf{r}, \omega-\omega_{0}\right)= & M(\omega) E_{0}\left(z, \omega-\omega_{0}\right) \exp \left[i \phi_{\mathrm{str}}(\omega)\right] \\
= & M(\omega) F(x, y) A_{0}\left(z, \omega-\omega_{0}\right) \\
& \times \exp \left(i k_{0} z\right) \exp \left[i \phi_{\mathrm{str}}(\omega)\right],
\end{aligned}
$$

where $A_{0}\left(z, \omega-\omega_{0}\right)$ is the input spectral amplitude. An inverse Fourier transform gives the field distribution leaving the stretcher, $E_{\mathrm{str}}(\mathbf{r}, t)=$ $A_{\text {str }}(z, t) F(x, y) \exp \left(i k_{0} z\right)$.

One can treat nonlinear propagation through the laser system by solving the level population equations coupled with the nonlinear Schrödinger equation. To concentrate on the effect of self-phase modulation only, we will neglect the effect of saturated gain. This assumption is satisfied even in saturated systems when the population inversion is depleted slowly over many passes (e.g., regenerative amplification). With saturation neglected, the amplifier chain can be treated with an effective net gain coefficient, $\alpha(\omega) L=\ln G(\omega)$, where $G(\omega)$ is the frequency-dependent net gain. Propagation through the amplifier chain then is described by the generalized nonlinear Schrödinger equation ${ }^{11}$

$$
\begin{aligned}
i \frac{\partial A}{\partial z}= & i \frac{\alpha}{2} A(z, t)+\frac{1}{2} \beta_{2} \frac{\partial^{2} A}{\partial \tau^{2}} \\
& -\frac{1}{6} \beta_{3} \frac{\partial^{3} A}{\partial \tau^{3}}-\gamma|A|^{2} A(z, t),
\end{aligned}
$$

where $\beta_{2}=\left(\partial^{2} k / \partial \omega^{2}\right) \omega_{0}$ represents dispersion of the group velocity $v_{g}, \beta_{3}=\left(\partial^{3} k / \partial \omega^{3}\right) \omega_{0}$ represents groupdelay dispersion, $\gamma$ is related to the nonlinear refractive index $n_{2}$ by the relation $\gamma=n_{2} \omega_{0} / c$, and $\tau=t-z / v_{g}$.

In the absence of the nonlinearity, $\gamma=0$, Eq. (3) is readily solved by a Fourier transform, with the result that the field leaving the amplifier chain is $A\left(z, \omega-\omega_{0}\right)=A_{0}\left(z_{\text {str }}, \omega-\omega_{0}\right) G(\omega) M(\omega)$ $\exp \left[i \phi_{\mathrm{str}}(\omega)\right] \exp \left[i \phi_{\mathrm{mat}}(z, \omega)\right]$, where $z_{\text {str }}$ is the position of the field leaving the stretcher. Material dispersion appears simply as a frequency-dependent phase function, $\phi_{\text {mat }}(z, \omega)=\beta_{2}\left(\omega-\omega_{0}\right)^{2} z / 2+\beta_{3}(\omega-$ 
$\left.\omega_{0}\right)^{3} z / 6+\ldots$ Propagation through the gratingpair pulse compressor is accomplished by multiplication by the phase function of the compressor, $\exp \left[i \phi_{\text {com }}(\omega)\right]$. If the compressor can be set exactly to cancel the dispersion of the stretcher and material, $\delta=\phi_{\text {com }}(\omega)+\left[\phi_{\text {str }}(\omega)+\phi_{\text {mat }}(z, \omega)\right]=0$, then transform-limited pulses are obtained.

The presence of self-phase modulation represented by the term $\gamma|A|^{2} A(z, t)$ in Eq. (3) makes it impossible to obtain transform-limited pulses on compression. In general, Eq. (3) must be solved numerically. However, given the practical limit that dispersion of the pulse owing to the initial pulse stretcher is much greater than the material dispersion in the amplifier chain, the propagation equation can be solved by a lumped-element or perturbative method. We initially neglect the material dispersion term in the nonlinear equation and then account for it in a second element containing no nonlinearity. This approach is valid for most CPA systems, in which an initial $\approx 100$-fs pulse is stretched by a factor of several thousand $\left(\tau_{\mathrm{str}} \approx 200-500 \mathrm{ps}\right)$ by the grating stretcher. In the absence of material dispersion $\left(\beta_{2}=\beta_{3}=0\right)$, Eq. (3) can be separated into two independent equations for the amplitude, $A$, and the phase, $\phi,\{A(z, t)=$ $\mathcal{A}(z, t) \exp [i \phi(z, t)]\}:$

$$
\frac{\partial \mathcal{A}}{\partial z}=\frac{\alpha}{2} \mathcal{A}(z, t), \quad \frac{\partial \phi}{\partial z}=\gamma A^{2} .
$$

The field leaving an amplifier of length $z$ is given by $A(z, t)=A(0, t) \exp (\alpha z / 2) \exp [i B(z, t)]$, where $A(0, t)$ is the field entering the amplifier, and, following Eq. (1), $B(z, t)=\left\{\gamma A^{2}(0, t)[\exp (\alpha z)-1] / \alpha\right\}$.

We find the field leaving the amplifier system, $A_{\text {sys }}(L, t)$, by summing the effect of each amplifier stage, adjusting for factors such as beam expansion and loss between stages. The small amount of material dispersion is accounted for by multiplication of the Fourier transform of $A_{\mathrm{sys}}(L, t)$ by the material dispersive phase, $\exp \left[i \phi_{\text {mat }}(L, \omega)\right]$, described above. We then obtain the compressed pulse by multiplying the result by $\exp \left[i \phi_{\text {com }}(\omega)\right]$ and calculating the Fourier transform.

The results of our calculations for an initial $\tau_{p}=$ $100 \mathrm{fs}$ Gaussian pulse, $A_{0}(0, t)=\exp \left[-2 \ln 2\left(t / \tau_{p}\right)^{2}\right]$, stretched to 200 ps before amplification and then recompressed with the idealized stretcher/compressor $(\delta=0)$, are shown in Fig. 1. For the case of $B=0$, the compressed pulse is transform limited and recompressed to its original shape and duration. As the pulse is amplified $(B>0)$, the recompressed pulse rapidly develops extended wings and a decrease in peak intensity. Even with a small, nonlinear phase shift $(B=1)$, the compressed pulse is seriously degraded, producing temporal wings that extend hundreds of femtoseconds before the peak of the pulse. Terawatt-class Ti:sapphire-based systems typically will exhibit a design-dependent $B$ integral between 1 and 3.

To observe this pulse broadening, we examined the autocorrelation of the recompressed pulse as a function of nonlinear phase in the regenerative amplifier of our Cr:LiSAF system. ${ }^{12}$ A $130-\mathrm{fs}, \operatorname{sech}^{2}\left(t / \tau_{p}\right)$ pulse is stretched to 400 ps by a grating pair and is injected into a $\mathrm{Cr}: \mathrm{LiSAF}$ ring regenerative amplifier. The pulse is amplified to the 5-20-mJ energy level, and the output of the regenerative amplifier is recompressed. By keeping the number of round trips in the cavity constant, and by varying the gain in the LiSAF amplifier rod, we can vary the energy fluence and, therefore, the total nonlinear phase $B$, without changing the amount of dispersive material traversed by the pulse. The $\mathrm{TEM}_{00}$ character of the regenerative amplifier cavity ensures a smooth beam and minimizes any uncertainty in the laser fluence and single-shot autocorrelation measurement. The regenerative amplifier was run at a low repetition rate for these experiments to eliminate any issues of thermal focusing.

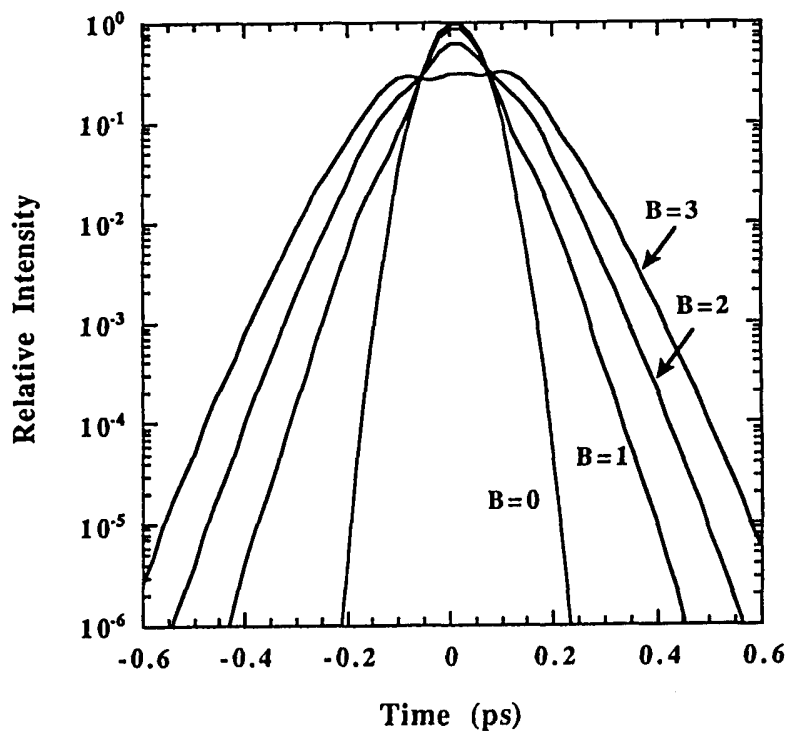

Fig. 1. Numerically calculated recompressed pulse shapes for an initially 100-fs Gaussian pulse, stretched to $200 \mathrm{ps}$, amplified to a level of $B=0,1,2$, or 3 .

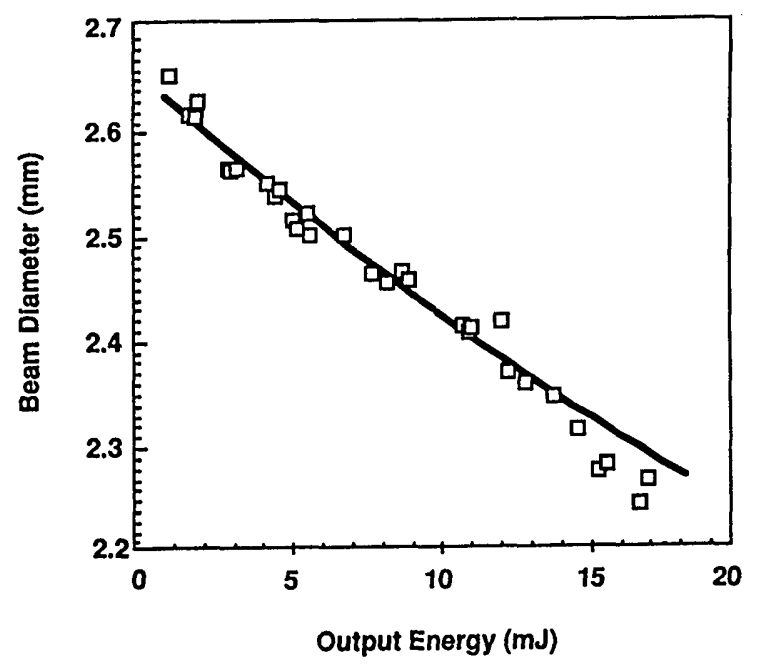

Fig. 2. Measurement of the Gaussian beam size $230 \mathrm{~cm}$ from the waist of the Cr:LiSAF regenerative amplifier cavity as a function of output energy. The solid curve is the beam size predicted by whole beam self-focusing inside the TEM 00 cavity. 


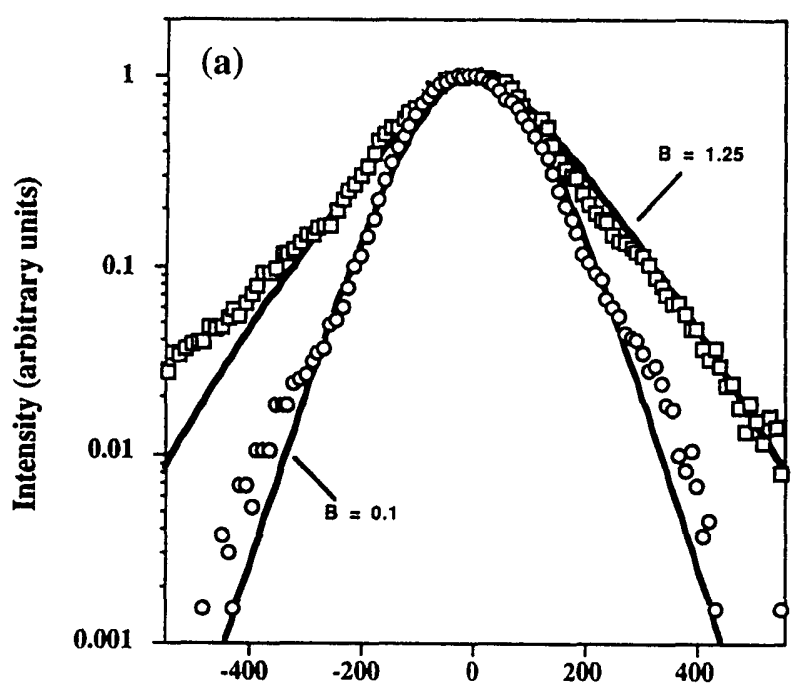

Time (fs)

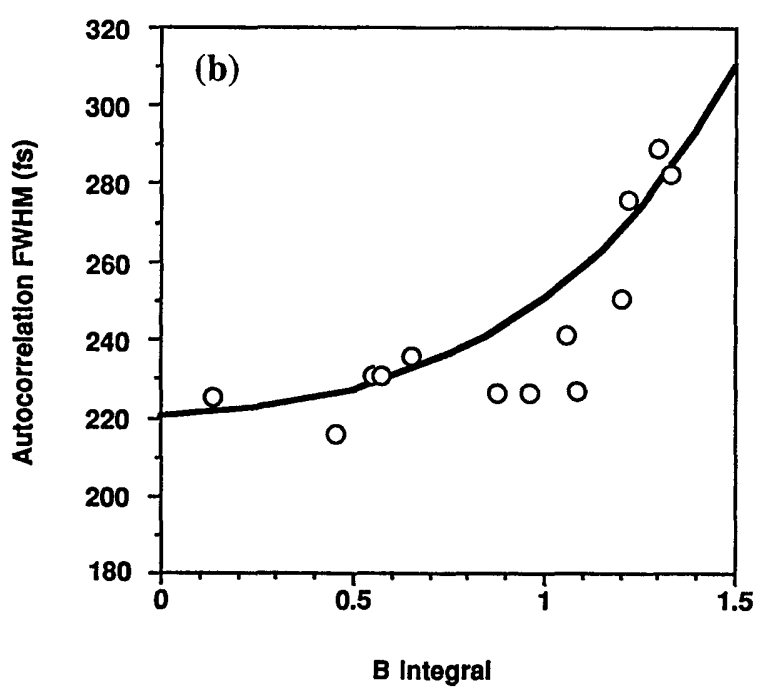

Fig. 3. (a) Comparison of the measured autocorrelation profile with our model calculation (solid curves) for two different values of the measured peak $B$ integral. (b) Comparison of the measured autocorrelation FWHM with the calculated width as a function of the accumulated $B$ integral.

The accumulated nonlinear phase was measured as a function of output energy by measurement of the whole-beam self-focusing of the Gaussian beam leaving the regenerative amplifier. The measured $1 / e^{2}$ diameter of the beam as a function of output energy is shown in Fig. 2. Our cavity model is shown to fit to these data points (solid curve) with a single adjustable parameter, the $n_{2}$ of Cr:LiSAF. The calculation shown in Fig. 2 uses a value of $n_{2}=4.8 \times 10^{-14}$ esu $\left(=1.4 \times 10^{-16} \mathrm{~cm}^{2} / \mathrm{W}\right)$, nearly a factor of 3 higher than a previous measurement. ${ }^{13}$

Second-order intensity autocorrelations of the recompressed, amplified pulse at low and high output energy are shown in Fig. 3(a), both normalized to a peak value of unity. One curve is the autocorrelation at $1.5 \mathrm{~mJ}(B \approx 0.1)$ representing a near-transform- limited $140-\mathrm{fs}$ pulse. The second curve is an autocorrelation trace at $16.9 \mathrm{~mJ}(B \approx 1.3)$. The broadening of the pulse clearly is evident, as is severe degradation of the pulse contrast. Autocorrelations calculated from the model described previously, and using the value of $n_{2}$ determined from the selffocusing measurements, also are shown on this plot as solid lines, in good agreement with the experimental data. The calculated autocorrelation (FWHM) is compared with the measured values as a function of the nonlinear phase in Fig. 3(b). The measured pulse width begins to broaden noticeably with $B \geq 1$.

We observe negligible modification of the pulse spectrum with the increasing width of the autocorrelation. This is because the frequency modulation resulting from self-phase modulation is determined by the time derivative of the nonlinear phase, $\omega(t)=$ $\omega_{0}(t)-\partial B / \partial t$. The fractional increase in bandwidth for a Gaussian pulse is approximately $\Delta \nu / \Delta \nu_{o} \approx$ $\Delta B / 2 \pi^{*}\left(\tau_{p} / \tau_{\mathrm{str}}\right)$. For a typical stretching ratio of 2000 for 100 -fs pulses, the spectral modulation will be of the order of $10^{-4}$.

In conclusion, self-phase modulation in the amplifier chain can degrade the quality of the compressed pulse in CPA systems, ultimately limiting the achievable pulse contrast. Even for modest values of the accumulated nonlinear phase, $B \leq 1$, significant energy can be removed from the central peak of the pulse and appear as a pedestal, thereby severely degrading pulse contrast and lowering the peak power.

This work was performed for the U.S. Department of Energy under the auspices of contract W-7405Eng-38.

\section{References}

1. P. Maine, D. Strickland, P. Bado, M. Pessot, and G. Mourou, IEEE J. Quantum Electron. 24, 398 (1988).

2. M. D. Perry and G. Mourou, Science 264, 917 (1994).

3. O. E. Martinez, IEEE J. Quantum Electron. QE23, 59 (1987).

4. M. Pessot, P. Maine, and G. Mourou, Opt. Commun. 62, 419 (1987).

5. E. B. Treacy, IEEE J. Quantum Electron. QE-5, 454 (1969).

6. M. D. Perry, F. G. Patterson, and J. Weston, Opt. Lett. 15, 381 (1990).

7. Y. H. Chuang, L. Zheng, and D. D. Meyerhofer, IEEE J. Quantum Electron. 29, 270 (1993).

8. R. L. Fork, C. H. Brito Cruz, P. C. Becker, and C. V. Shank, Opt. Lett. 12, 483 (1987).

9. W. E. White, A. Sullivan, and F. G. Patterson, Opt. Lett. 18, 1343 (1993); P. Tournois, Electron. Lett. 29, 1414 (1993).

10. B. E. Lemoff and C. P. Barty, Opt. Lett. 18, 1651 (1993).

11. I. Mercer, Ph.D. dissertation (University of London, 1993).

12. T. Ditmire, H. Nguyen, and M. D. Perry, J. Opt. Soc. Am. B 11, 580 (1994).

13. M. Richardson, M. J. Soileau, E. Van Stryland, P. Beaud, Y. F. Chen, R. DeSalvo, S. Garnov, D. Hagan, S. Klimentov, and B. Chai, Proc. Soc. Photo-Opt. Instrum. Eng. 1848, 392 (1993). 